\title{
Visualization of localized perturbations on a (001) surface of the ferromagnetic semimetal $\mathrm{EuB}_{6}$
}

\author{
Sahana Rößler $\odot,{ }^{1, *}$ Lin Jiao $\odot,{ }^{1, \dagger}$ Silvia Seiro $\odot,{ }^{1, \ddagger}$ Priscila F. S. Rosa $\odot,{ }^{2,3}$ Zachary Fisk $\odot,{ }^{2}$ \\ Ulrich K. Rößler $\odot,{ }^{4}$ and Steffen Wirth $\oplus^{1, \S}$ \\ ${ }^{1}$ Max Planck Institute for Chemical Physics of Solids, Nöthnitzer Straße 40, 01187 Dresden, Germany \\ ${ }^{2}$ Department of Physics, University of California, Irvine, California 92697, USA \\ ${ }^{3}$ Condensed Matter and Magnet Science Group, Los Alamos National Laboratory, Los Alamos, New Mexico 87545, USA \\ ${ }^{4}$ IFW Dresden, Helmholtzstraße. 20, 01069 Dresden, Germany
}

(Received 18 November 2019; revised manuscript received 11 May 2020; accepted 13 May 2020; published 11 June 2020)

\begin{abstract}
We performed scanning tunneling microscopy (STM) and spectroscopy on a (001) surface of the ferromagnetic semimetal $\mathrm{EuB}_{6}$. Large-amplitude oscillations emanating from the elastic scattering of electrons by the surface impurities are observed in topography and in differential conductance maps. Fourier transform of the conductance maps embracing these regions indicate a holelike dispersion centered around the $\bar{\Gamma}$ point of the two-dimensional Brillouin zone. Using density functional theory slab calculations, we identify a spin-split surface state, which stems from the dangling $p_{z}$ orbitals of the apical boron atom. Hybridization with bulk electronic states leads to a resonance enhancement in certain regions around the $\bar{\Gamma}$ point, contributing to the remarkably strong real-space response around static point defects, which are observed in STM measurements.
\end{abstract}

DOI: 10.1103/PhysRevB.101.235421

\section{INTRODUCTION}

Rare-earth hexaborides are not only technologically important as hot cathode materials owing to their low work functions [1], but they also raise fundamental interest due to their diverse and exotic properties. Albeit all rare-earth hexaborides crystallize in the cubic $\mathrm{CaB}_{6}$ structure type, they exhibit distinct electronic properties, in part due to different $f$-shell electron occupation. While $\mathrm{CeB}_{6}$ exhibits a multipolar order [2], $\mathrm{YB}_{6}$ displays superconductivity below about $7.2 \mathrm{~K}$ [3]. $\mathrm{EuB}_{6}$ is a ferromagnetic semimetal for which the concept of magnetic polarons was long discussed [4-7] as the underlying mechanism for its colossal magnetoresistance (CMR) behavior. $\mathrm{SmB}_{6}$ was proposed to be the first topological Kondo insulator $[8,9]$ and has been intensively investigated in the past decade.

$\mathrm{EuB}_{6}$ is ferromagnetic below the transition temperature $T_{c 2}=12.6 \mathrm{~K}[10,11]$. At slightly higher temperatures, a metal-semimetal transition takes place, with a drastic drop of the resistivity below $T_{c 1}=15.3 \mathrm{~K}$ in zero field [10-12].

\footnotetext{
*roessler@cpfs.mpg.de

${ }^{\dagger}$ Present address: Department of Physics and Frederick Seitz Materials Research Laboratory, University of Illinois Urbana-Champaign, Urbana, Illinois 61801, USA.

${ }^{\ddagger}$ Present address: IFW Dresden, Helmholtzstraße. 20, 01069 Dresden, Germany.

§wirth@cpfs.mpg.de
}

Published by the American Physical Society under the terms of the Creative Commons Attribution 4.0 International license. Further distribution of this work must maintain attribution to the author(s) and the published article's title, journal citation, and DOI. Open access publication funded by the Max Planck Society.
In presence of an external magnetic field, a large negative magnetoresistance (MR) is found, which is strongest around $T_{c 1}[11-13]$. This CMR is related to a percolation-type transition resulting from the overlap of magnetic polarons, which causes a delocalization of the hole carriers $[5,11,13]$ (see, e.g., Ref. [14] for a brief review). Existence of such polarons above $T_{c 1}$ was also confirmed by several spectroscopic techniques $[12,15,16]$, including scanning tunneling spectroscopy (STS) [7], which visualized nanoscale clusters of enhanced local conductance even above $T_{c 1}$.

$\mathrm{EuB}_{6}$ is considered a semimetal with only a small overlap of valence and conduction bands centered at the $X$ point of the cubic Brillouin zone [17]. Quantum oscillation data were interpreted in this framework of $X$-point band overlap $[18,19]$. This interpretation was contested based on the results of angle-resolved photoemission spectroscopy (ARPES) measurements, which indicated an $X$-point band gap of $1 \mathrm{eV}$ (i.e., no overlap between the valence and the conduction bands) with the bulk Fermi level $\left(\epsilon_{F}\right)$ near the bottom of the conduction band $[20,21]$. In addition, band structure calculations in the ferromagnetic state predicted $\mathrm{EuB}_{6}$ to be a half-metallic semimetal with complete spin polarization at $E_{\mathrm{F}}[22,23]$. Andreev reflection spectroscopy [24], however, indicated that only the valence band is spin polarized. Further, (001) surfaces of hexaborides are polar. This polarity is expected to render various types of surface reconstructions as witnessed in the scanning tunneling microscopy (STM) measurements on $\mathrm{SmB}_{6}$ (001) surfaces [25-32]. In $\mathrm{EuB}_{6}$, the formation of a two-dimensional (2D) electron gas confined to the surface was first reported by Denlinger et al. [21,33] from the observation of temporal changes in the ARPES spectra. These experiments indicated that the position of the chemical potential can vary with cleave, surface location, and time [21]. Here, using STM, we visualize a formation of rings due to 
localized perturbations caused by defects on the (001) surface of $\mathrm{EuB}_{6}$. From our combined study of STM/S and density functional theory (DFT) calculations, we show that the rings are formed due to the screening of the impurities by a holelike bulk band, and its strength is enhanced where the electronlike surface state dispersion crosses into the surface-projected bulk states.

\section{EXPERIMENT}

The single crystals of $\mathrm{EuB}_{6}$ were grown using the $\mathrm{Al}$ flux method [34]. The samples were cleaved along the [001] direction in situ in the UHV chamber at a temperature of $\approx 20 \mathrm{~K}$. The STM measurements were performed in the temperature range of $0.35-100 \mathrm{~K}$. To address the surface-electronic structure of $\mathrm{EuB}_{6}$ we performed DFT calculations considering spin-split bands for periodic supercells in the forms of slabs terminating on both surfaces in $\mathrm{B}_{6}$ octahedra, in concert with our experiments reported below. The calculations have been done with the full-potential local orbital (FPLO) approach [35] and using the generalized-gradient approximation (GGA) as the exchange-correlation functional [36].

\section{RESULTS AND DISCUSSION}

In Figs. 1(a) and 1(b), topographic images of atomically resolved, clean, and nonreconstructed (001) surfaces of $\mathrm{EuB}_{6}$ on areas of $10 \times 10 \mathrm{~nm}^{2}$ and $5 \times 5 \mathrm{~nm}^{2}$ are presented. The height scans along the lines shown in the topography are plotted in Figs. 1(c) and 1(d). The scan along [100] direction in Fig. 1(a) shows atoms placed at a distance of $\approx 4.18 \AA$, which is in agreement with the lattice parameter $a \approx 4.183 \AA$ of $\mathrm{EuB}_{6}$ [37]. No additional corrugations are visible along [110] direction in Fig. 1(a). However, a height scan along [110] direction, cyan line in Fig. 1(b), exhibits corrugations at a distance of $a / \sqrt{2}$. Similar topographies observed in $\mathrm{SmB}_{6}$ $[27,28]$ were assigned to B- and Sm-terminated surfaces, respectively. With this assignment, the corrugations observed at a distance of $a / \sqrt{2}$ in the height scan along [110] direction likely correspond to the apical $\mathrm{B}$ atoms of the $\mathrm{B}_{6}$ octahedra one layer below, and in between, the $\mathrm{Eu}$ atoms of the surface layer as seen in the crystal structure of $\mathrm{EuB}_{6}$, inset of Fig. 1(b). Based on the same argument, we conclude that the topography in Fig. 1(a) is consistent with B termination and that in Fig. 1(b) with Eu termination. However, the latter type of topographies were rarely observed [38]. Surprisingly, a topography encompassing an impurity on a B-terminated surface was found to be drastically different, as can be seen in topography Fig. 1(e). This image shows a circular wave of large amplitude, caused by the localized perturbation caused by the impurity potential. The tip displacements along the lines in Fig. 1(e) are presented in Fig. 1(f). Here, the tip displacement is a result of actual height changes and, more importantly, changes in the local density of states (DOS). From these height scans, the wavelength $\lambda$ of the oscillations are found to be $\approx 16.8 \AA$ at a bias voltage $V=-0.2 \mathrm{~V}$. The rings were also observed for positive bias voltages, but their amplitudes were found to be rather weak, see Appendix, Fig. 6. We traced the oscillations up to $80 \mathrm{~K}$ (see Appendix, Fig. 7). As can be seen from Fig. 7, the oscillations are
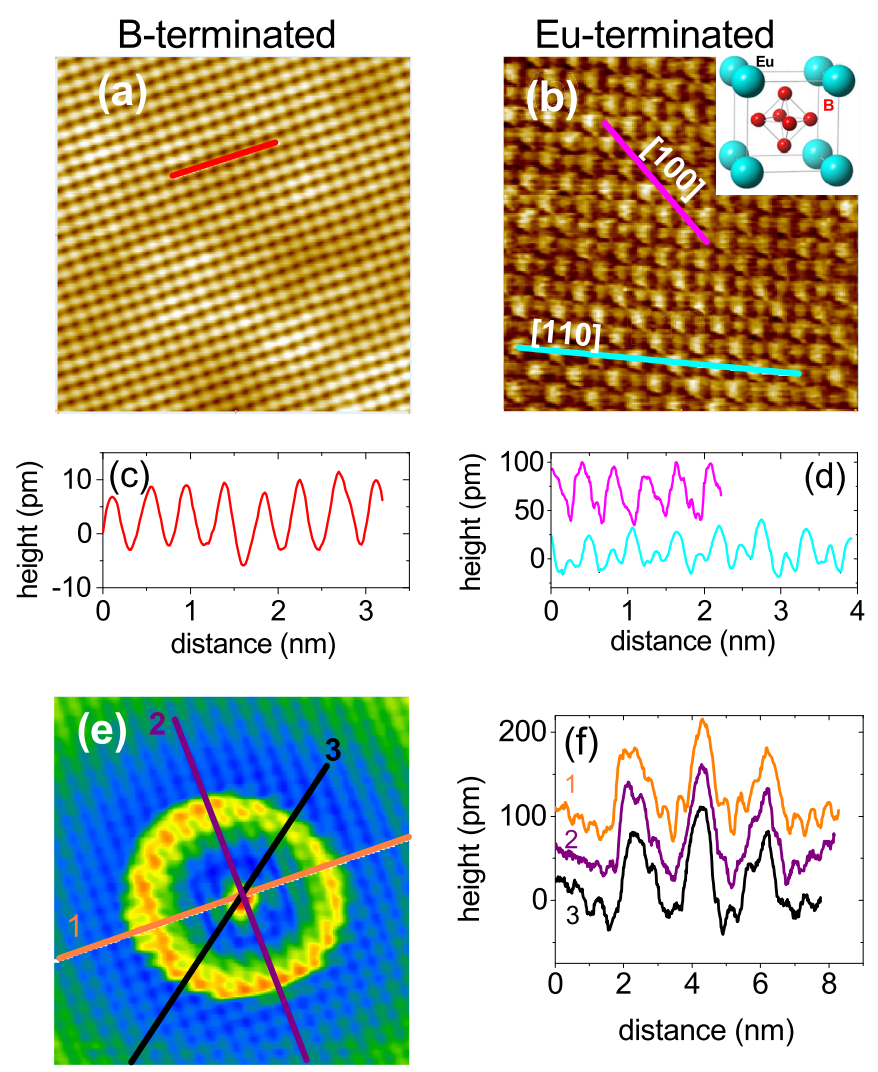

FIG. 1. Topographies of $\mathrm{EuB}_{6}$ taken at $T=6 \mathrm{~K}$ on clean areas showing atomic resolution of (a) B-terminated surface on an area of $10 \times 10 \mathrm{~nm}^{2}$ (b) Eu-terminated surface on an area of $5 \times 5 \mathrm{~nm}^{2}$. The topographies were taken with a bias voltage $V=+0.2 \mathrm{~V}$ and set-point current $I_{\mathrm{sp}}=500 \mathrm{pA}$. Inset: Crystal structure of $\mathrm{EuB}_{6}$. (c)-(d) Height profiles along the red, cyan, and pink lines in (a) and (b). (e) Topography on an area of $8 \times 8 \mathrm{~nm}^{2}$ around an impurity with $V=-0.2 \mathrm{~V}$ and $I_{\mathrm{sp}}=500 \mathrm{pA}$. The conductance maps $g(V, \vec{r})=$ $d I(V, \vec{r}) / d V$ on this topography is presented in Fig. 3. (f) Tip displacement $\left(\mathrm{t}_{\mathrm{dis}}\right)$ profiles along the lines 1,2 , and 3 in (e). The scans along the lines 1 and 2 are shifted up by $+100 \mathrm{pm}$ and $+50 \mathrm{pm}$ for clarity.

unaffected by the two phase transitions at $T_{c 1}$ and $T_{c 2}$, below which the resistivity drops more than an order of magnitude.

One of the mechanisms that can produce similar effects observed here is a tip-induced band bending (TIBB). It occurs in materials with low charge carriers as $\mathrm{EuB}_{6}$, in which the applied electric field can penetrate into the sample due to poor electric screening. These effects have been well investigated in semiconductors [39-41], lightly doped Mott insulators $[42,43]$, and topological insulators [44]. In these materials, the individual impurity can be charged by the bias voltage of the STM tip thereby increasing the local DOS available for tunneling. This effect produces charge compensation rings, or bubbles, around the impurities. The charge carrier concentrations $n$ in $\mathrm{EuB}_{6}$ estimated from quantum oscillation experiments were found to be $1.2 \times 10^{20} \mathrm{~cm}^{-3}$ for electrons and $1.01 \times 10^{20} \mathrm{~cm}^{-3}$ for holes, after considering spin polarization effects [19,24]. This value of $n$ is about three orders of magnitude lower than that in copper and about three orders of magnitude higher than that in the classical semimetal bismuth. Therefore, we checked for TIBB effects in our sample. The 

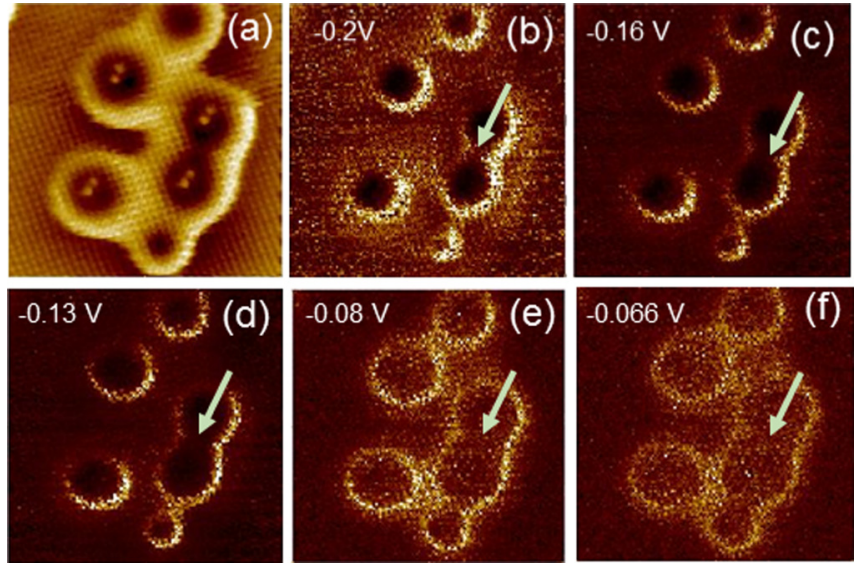

FIG. 2. (a) A topography on an area of $15 \mathrm{~nm} \times 15 \mathrm{~nm}$ containing several impurities. The image was taken with a bias voltage $V=-0.2 \mathrm{~V}$ and a tunneling current $I_{\mathrm{sp}}=50 \mathrm{pA}$. (b)-(f) $d I / d V$ maps on the area shown in (a) at different bias voltages indicated in the images. The bright region represent enhanced conductance caused by the screening effect. The arrows point at a region of overlap of two rings produced by different impurity centers. Lower conductance of this region suggests a destructive interference.

TIBB depends on the STM tip-sample distance $d$ : the smaller the distance, the larger the effect $[43,45]$. Since the tunneling current falls off exponentially with respect to $d$, we varied $d$ by changing the set point of the tunneling current $\left(I_{\mathrm{sp}}\right)$ by an order of magnitude and did not see any difference in the size of the rings (see Appendix, Figs. 8 and 9). This suggests that any TIBB effect, if present, is minimal in our samples. Further, the effect observed here is not due to a simple enhancement of the local DOS, rather due to a collective wavelike scattering phenomenon, which is further evidenced in Fig. 2, as described below.

In Fig. 2(a), a topographic image of an area of $15 \mathrm{~nm} \times$ $15 \mathrm{~nm}$ containing multiple impurities is presented. It can be seen that each impurity center produces its own scattering ring. In Figs. 2(b)-2(f), $d I / d V$ maps on the same area for different bias voltages are displayed. With increasing $V$ from $-0.2 \mathrm{~V}$ to $-0.066 \mathrm{~V}$, the size of the ring increases, which suggests a holelike dispersion of the underlying band structure in this energy range (see also below). Moreover, as the rings grow in size, they interfere with each other and produce a region of destructive interference; one example is marked by an arrow in Figs. 2(b)-2(f). If the formation of the rings was solely due to the enhancement of local DOS produced by TIBB, then, when two rings meet, an enhancement of the intensity is expected. The destructive interference observed here confirms that the rings are indeed caused by wavelike phenomena.

To directly probe the dispersion of the band causing the oscillation, we have performed $d I / d V$ maps on a topographic area containing a single impurity, shown in Fig. 1(e) in the bias voltage range $-0.2 \mathrm{~V}$ to $+0.2 \mathrm{~V}$ in intervals of $2 \mathrm{mV}$. The $d I(V) / d V$ curves typically observed on this kind of surfaces are presented in Appendix, Fig. 10(b). In Fig. 3(a), $d I / d V$ maps for selected bias voltages are displayed. The size of the rings remains the same up to $V \approx-144 \mathrm{mV}$, but begins to increase in the range $-144 \mathrm{mV}$ to $-68 \mathrm{mV}$ indicating a (a)

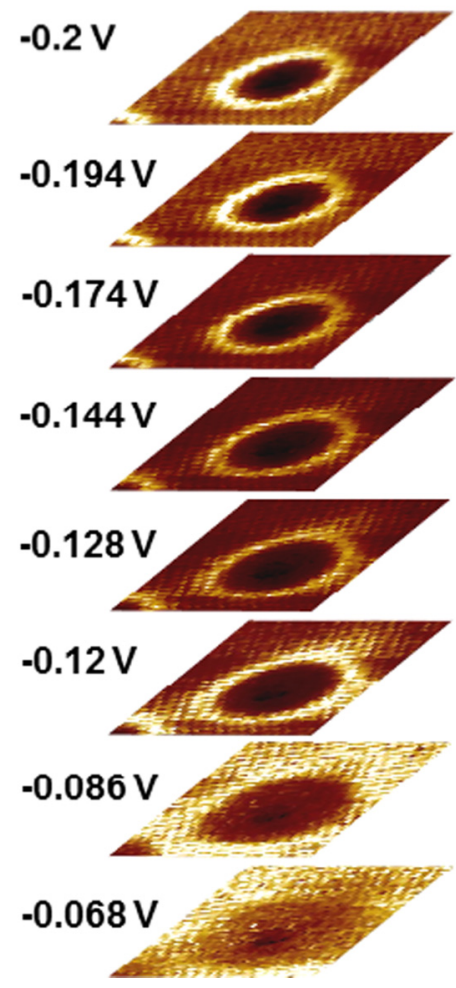

(b)

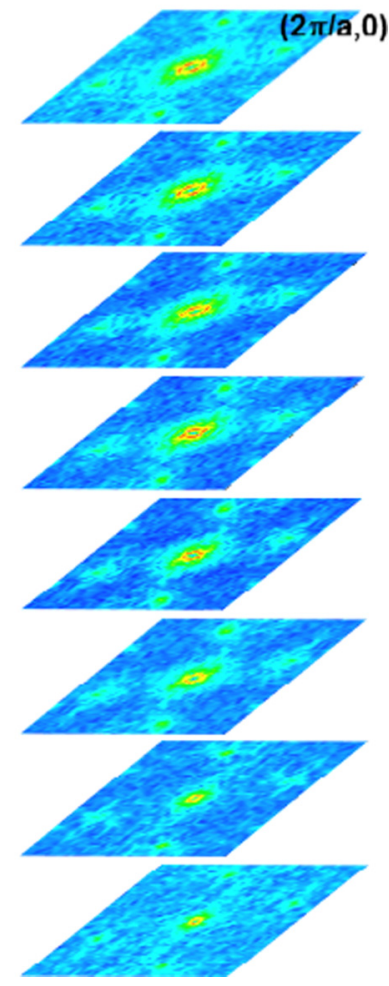

FIG. 3. (Left) $d I / d V$ maps on a surface area of $8 \times 8 \mathrm{~nm}^{2}$ displaying the scattering produced by a single impurity and (right) corresponding Fourier transforms. The maps were taken at different bias voltages as shown in the figure. The measurements were performed at $T=6 \mathrm{~K}$.

holelike dispersion, as mentioned earlier. The rings were not discernible in the $d I / d V$ maps for $V \geqslant-68 \mathrm{mV}$, indicating that the top of the hole band probed here is about $\approx 68 \mathrm{meV}$ below the Fermi level. Further, we were also not able to resolve the rings in the $d I / d V$ maps for the positive bias voltages, although rings with comparatively weak amplitude were found in the topography. A similar behavior was found in Si-doped GaAs [46]. To obtain the band dispersion, we performed the Fourier transforms of the $d I / d V$ maps, see Fig. 3(b). The four bright spots close to the sides' center correspond to Bragg peaks at $( \pm 2 \pi / a, 0),(0, \pm 2 \pi / a)$, one of them is marked in Fig. 3(b). From these maps, we obtained the band dispersion as a function of $q$. From the relation $\vec{q}=2 \vec{k}$, the $\vec{k}$ dependence of the band is obtained, see Fig. 4 .

The electronic surface structure of hexaborides has been investigated earlier by calculations [47-50]. In order to assign the oscillations on B-terminated surfaces to a particular band, we performed DFT calculations for $\mathrm{EuB}_{6}$ by considering spinsplit bands for periodic supercells in the form of slabs terminating on both surfaces in $\mathrm{B}_{6}$ octahedra, Fig. 5(a). The $4 f$ electrons of $\mathrm{Eu}^{2+}$ are included in a fully polarized $(S=7 / 2$, $L=0$ ) open core. This removes its half-filled $4 f$ shell from the $\epsilon_{F}$, but enables hybridization of $4 f$ and $5 d$ states on $\mathrm{Eu}$ and spin splitting of valence band states of B [51]. A surface state appears as an extra electronic band with width of about $0.3 \mathrm{eV}$ close to $\epsilon_{F}$, as seen from the DOS, Fig. 5(b). This band is mainly formed from contributions of the $2 p_{z}$ orbital at the 


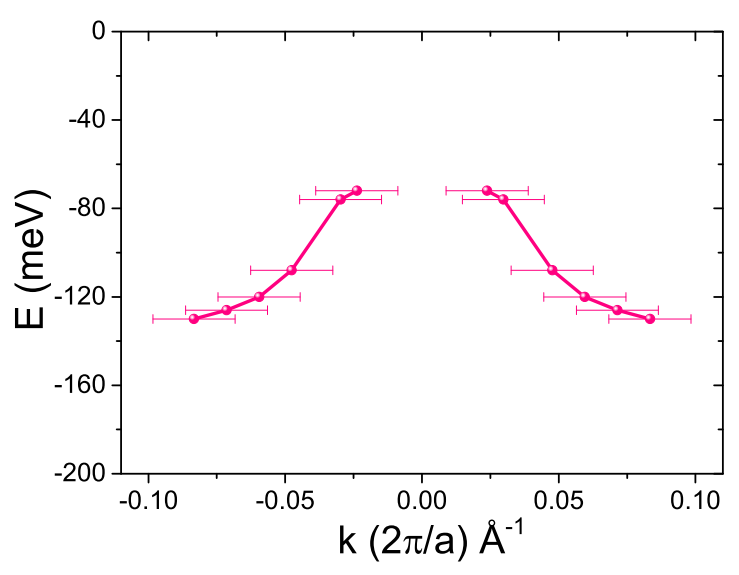

FIG. 4. E vs. $\vec{k}$ for the surface state plotted along $\overline{\mathrm{X}}-\bar{\Gamma}-\overline{\mathrm{X}}$ direction of the cubic Brillouin zone. The error bars represent the image resolution (pixel size) in Fig. 3(b).

apical first boron layer $\mathrm{B}_{(1)}$, but it has weights also from $2 p_{z}$ orbitals down to the fourth boron layer $\mathrm{B}_{(4)}$ and from $2 s$ states within these first four boron layers at the surface. Remarkably, the band is spin split by $\Delta_{s} \simeq 0.6 \mathrm{eV}$. Figure 5(c) gives an overview of the band structure demonstrating the dispersion of the surface state in the Brillouin zone (BZ) of the 2D periodic slab. As can be seen from the Appendix, Fig. 11, the surface state hybridizes with the bulk electronic structure, particularly around the $\bar{\Gamma}$ point, which leads to a continuum of extended states with resonant enhancement near the surface.

(a)
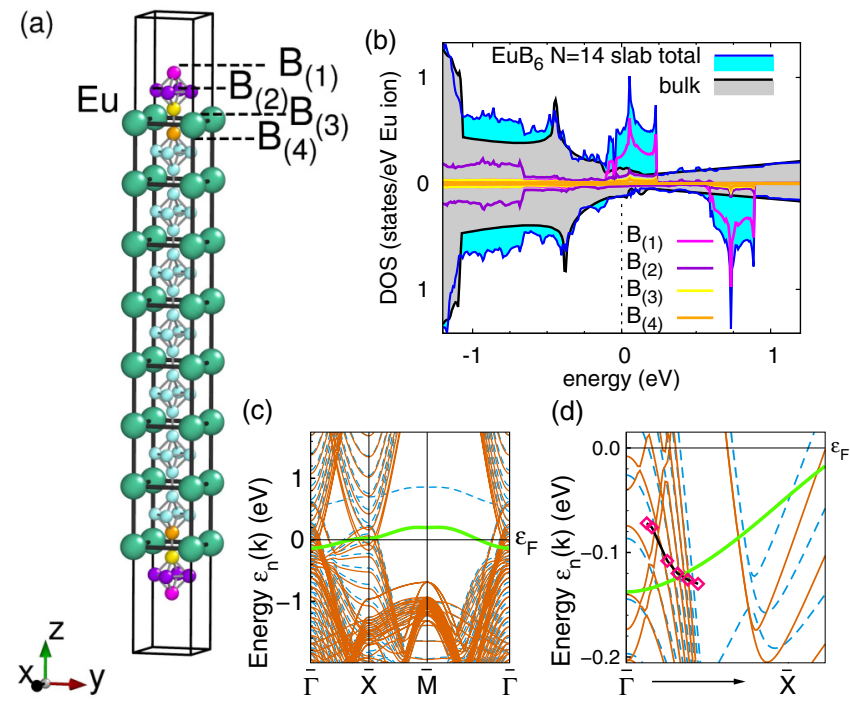

FIG. 5. (a) Unit supercell of a slab with $B_{6}$ termination on both surfaces, $\mathrm{Eu}_{N} \mathrm{~B}_{(N+1) 6}$, here with $N=8$, employed as model for the electronic surface structure. The first four boron layers are marked at the top, $\mathrm{B}_{(i)}, i=1, \ldots, 4$. (b) Comparison of electronic density of states (DOS) between bulk electronic states and a $N=14$ slab. Spin majority/minority DOS are shown on upper/lower ordinate. Also shown, projected partial DOSs for $B_{(1)}$ to $B_{(4)}$. (c) and (d) Spin-split band structure as projection on the two-dimensional $\mathrm{BZ}$ for the $x y$-periodic square lattice of the (001) surface. Majority (minority) spin bands are shown by solid red (dashed blue) lines, the surface spin-majority state by a thicker green line. In (d) the diamonds depict the dispersion of the experiment, from Fig. 4.
In the slab calculations, this continuum of resonant states is represented by a dense set of electronic bands, see cut from $\bar{\Gamma}$ to $\bar{X}$ in Figs. 5(c) and 5(d). The corresponding electronic states consist of mixed contributions from orbitals in the interior of the slab and from the terminating $\mathrm{B}_{6}$ octahedra. These surfaceenhanced resonant states are expected to contribute the major part of the electronic screening cloud around point defects at the surface. We note that the calculations indicate a negligible angular dependence of the dispersion of the projected bulk bands, in line with the observed isotropic shape of the rings. The experimental dispersion of the states contributing to the oscillations is included in Fig. 5(d). These electronic states correspond to an energy and wave-vector range along $\bar{\Gamma}-\overline{\mathrm{X}}$, which is close to the region where the proper surface state hybridizes with the continuum of bulk states.

Thus, the oscillations seen in experiment are related to surface-enhanced bulk occupied states, which are resonantly hybridized with the surface state. This could be also the reason why we only see weak oscillations for the positive bias voltages, at which unoccupied states are probed. The good match between the surface energy level, the location of bulk continuum of enhanced states and the experimental dispersion may be fortuitous. Its exact location and the dispersion of these resonances in the band structure are determined by details like relaxation of the surface structure (not considered in our calculation) and the band filling of the surface states, which depend on impurity concentration and even the size of the $\mathrm{B}_{6}$-terminated domains. However, from our calculations we see that the spin splitting is necessary to push down the majority spin-surface state sufficiently to gain appreciable filling of this band and give rise to resonant electronic states at about $0.1 \mathrm{eV}$ below $\epsilon_{F}$. For the surface states, owing to its $2 \mathrm{D}$ nature, the DOS at $\epsilon_{F}$ is large and can drive the spin splitting by the Stoner mechanism for itinerant ferromagnetism. The spin-majority band is pushed down in energy and acquires a fractional filling. Using Stoner theory, a Curie temperature for magnetic long-range order would be $T_{C}^{s} \sim \Delta_{s}=0.6 \mathrm{eV}$ $\gg 300 \mathrm{~K}$. Naturally, long-period fluctuations are expected to impede ferromagnetic long-range order at the surface. But, the size of $\Delta_{s}$ suggests that the electronic structure locally remains in a spin-split state towards higher temperature, similarly to any itinerant metallic paramagnet. Moreover in $\mathrm{EuB}_{6}$, the exchange field provided by the spin-polarized $\mathrm{Eu}$ $5 d$ orbitals and the anisotropy stemming from the polar nature of the surface could defeat the prohibition of $2 \mathrm{D}$ ordering by the Mermin-Wagner theorem. These observations suggest that the electronic structure at the surface is effectively spin split up to large temperatures.

\section{CONCLUSIONS}

In conclusion, using STM/S, we have shown localized perturbations caused by impurities on the (001) surface of $\mathrm{EuB}_{6}$. From the Fourier analysis of scattering produced by a single surface impurity, we detected a band with a holelike dispersion, lying $\approx 68 \mathrm{meV}$ below the Fermi level. Using DFT slab calculations, we show the presence of a spin-split surface state band hybridizing with the bulk states close to the $\bar{\Gamma}$ point. The large amplitude oscillations are attributed to the electronic screening effect in the holelike bulk states and its strength 
is enhanced where the electronlike surface state dispersion crosses into or in resonance with the those surface-projected bulk states.

\section{ACKNOWLEDGMENTS}

We thank J. W. Allen, J. D. Denlinger, M. Richter, and M. Wenderoth for enlightening discussions, and U. Nitzsche for support to the computational work. Financial support from the Deutsche Forschungsgemeinschaft (DFG) within the Schwerpunktprogramm SPP1666 is gratefully acknowledged. L.J. thanks the Alexander-von-Humboldt foundation for financial support. Work at Los Alamos National Laboratory was performed under the auspices of the U.S. Department of Energy, Office of Basic Energy Sciences, Division of Materials Sciences and Engineering.

\section{APPENDIX}

In Fig. 6, topographic images of the same area obtained in a dual bias mode are presented. In this mode, the bias voltage can be switched between different values for forward and backward scanning. For the image in Fig. 6(a), a negative voltage $V=-0.2 \mathrm{~V}$ and Fig. $6(\mathrm{~b})$, and a positive $V=+0.2 \mathrm{~V}$ was used. Hence, a spatial information about the unoccupied and occupied states can be obtained for the same area of the topography. In Fig. 6(c), the tip-displacement scans along the red and blue lines in Figs. 6(a) and 6(b) are plotted. Although the value of the height of the impurity in Fig. 6(b) appears to be higher, the waves emanating from the impurity site have
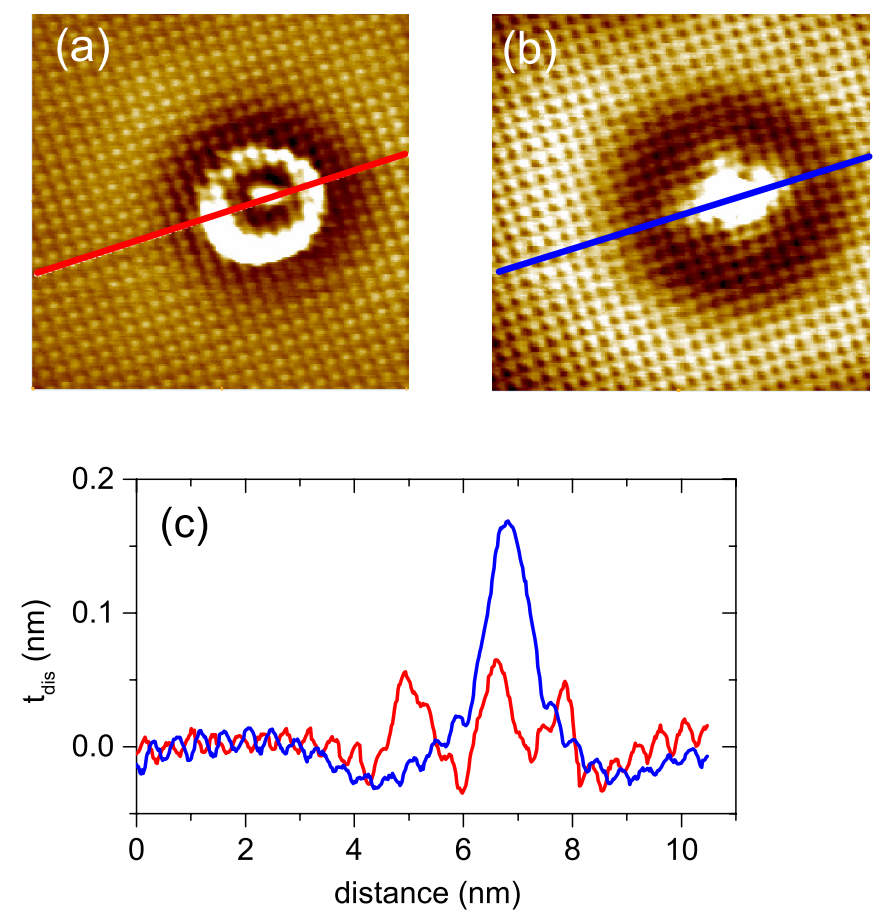

FIG. 6. (a) STM topographies on a same area around an impurity taken on an area of $10 \times 10 \mathrm{~nm}^{2}$ with a tunneling current $I_{\mathrm{sp}}=$ $500 \mathrm{pA}$ in a dual bias mode with bias voltages of (a) $V=-0.2 \mathrm{~V}$ and (b) $+0.2 \mathrm{~V}$, respectively. (c) Tip displacement $\left(\mathrm{t}_{\mathrm{dis}}\right)$ profiles along the red and blue lines in (a) and (b), respectively.
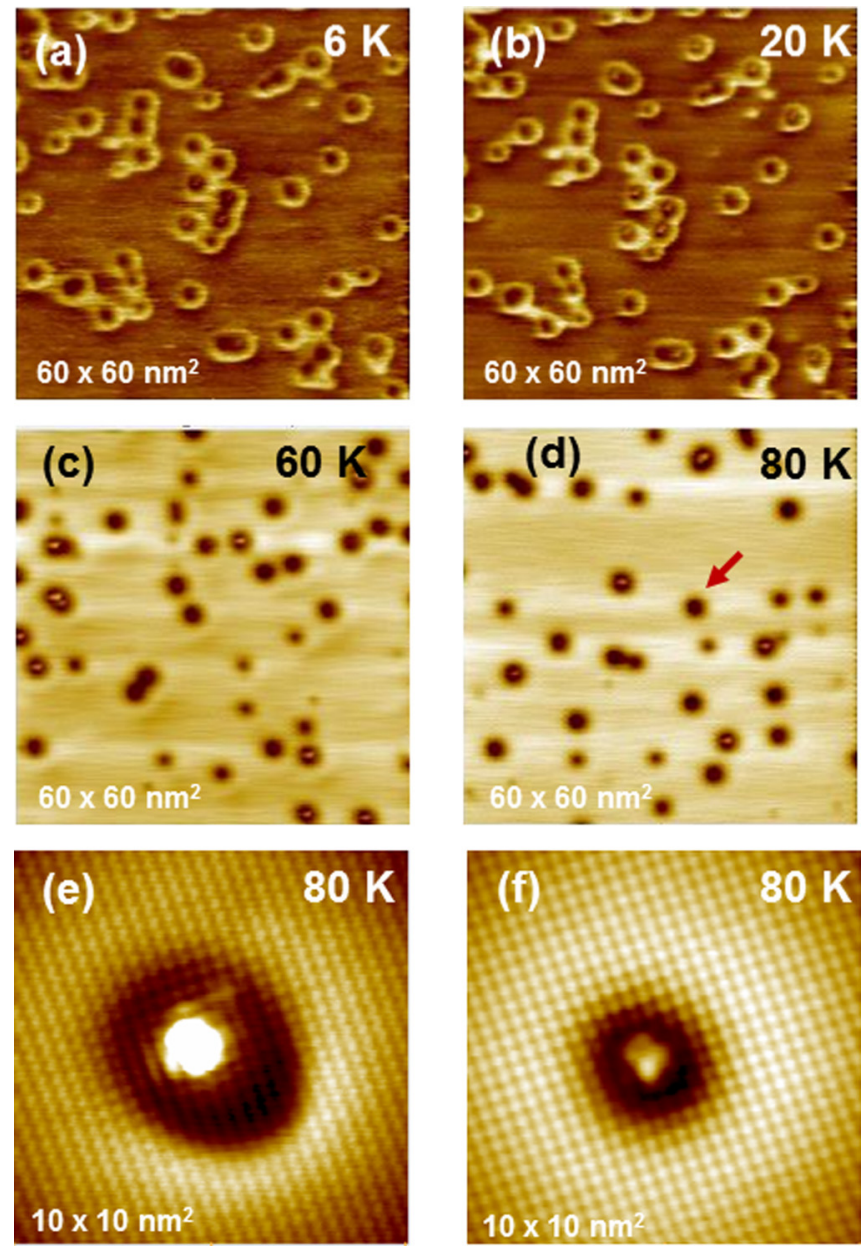

FIG. 7. (a)-(d) STM topographies of $\mathrm{EuB}_{6}$ taken at different temperatures ranging from $6-80 \mathrm{~K}$ on a surface area of $60 \times 60 \mathrm{~nm}^{2}$. The images at $6 \mathrm{~K}$ and $20 \mathrm{~K}$ were taken nearly on the same area. The bias voltage and the tunneling current were set at $V=-0.2 \mathrm{~V}$ and $I_{\mathrm{sp}}=500 \mathrm{pA}$, respectively, for all four images. (e) and (f) A zoom-in of an area of $10 \times 10 \mathrm{~nm}^{2}$ around an impurity in (d), pointed by a red arrow. The topographies were taken in a dual bias mode with $V=+0.2 \mathrm{~V}(\mathrm{e})$ and $-0.2 \mathrm{~V}(\mathrm{f}) . I_{\mathrm{sp}}=500 \mathrm{pA}$.

lower amplitude when compared to those in Fig. 6(a), i.e., the rings appear weaker in the unoccupied part of the band structure.

In Fig. 7(a)-7(d) we present topographies on an area of $60 \times 60 \mathrm{~nm}^{2}$ of a B-terminated surface containing several impurity sites taken at different temperatures. The images at $T=6 \mathrm{~K}$ and $20 \mathrm{~K}$ are below and above the temperatures of the phase transition, respectively. The topographies taken at these temperatures are nearly on the same location and they do not display any difference. We measured topographies at several temperatures up to $80 \mathrm{~K}$ and observed rings, see Fig. 7(e) and 7(f) for high-resolution topographies taken at $80 \mathrm{~K}$ in dual bias mode. This result implies that the oscillations are unaffected by the two phase transitions at $T_{c 1}$ and $T_{c 2}$, below which the resistivity drops more than an order of magnitude. The ferromagnetic ordering mainly affects the band structure at the $X$ point and is believed to be responsible for the polaronic behavior [7]. Our results indicate that effects observed here are unrelated to the $X$-point band structure. 

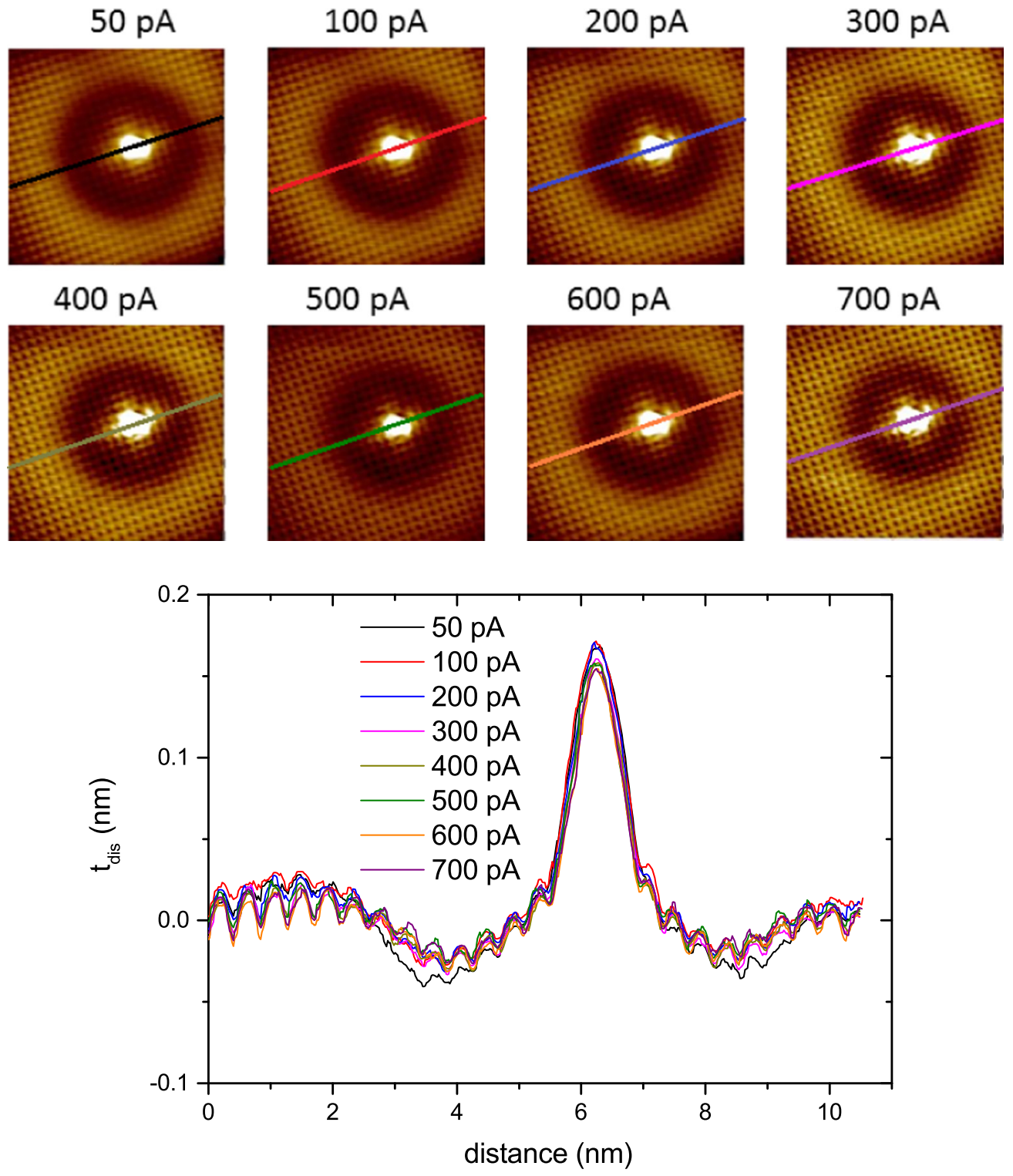

FIG. 8. STM topographies of $\mathrm{EuB}_{6}$ (top and middle row) taken on a surface area of $10 \times 10 \mathrm{~nm}^{2}$ at different tunneling currents, $I_{\mathrm{sp}}$. The bias voltage $V=+0.2 \mathrm{~V}$. In the graph (bottom) tip displacement $\left(\mathrm{t}_{\mathrm{dis}}\right)$ profiles along the lines in the topographies are shown. Note that, for a given $I_{\mathrm{sp}}$, same color is used for the lines in the topography and line profiles in the graph. The small corrugations of about $20 \mathrm{pm}$ in height reflect the lattice constant $a=4.18 \AA$.

Indeed, we show in the main text, using density functional theory (DFT) calculations that the oscillations observed here are arising due to the hybridization of the holelike bulk band lying about $68 \mathrm{meV}$ below the Fermi level with the surface state close to the $\Gamma$ point.
In Figs. 8 and 9, we present topographic images on two different areas (and likely different types of impurities) taken at different current set points, $I_{\mathrm{sp}}$. The bottom panel of Fig. 8 shows the line profiles along the lines drawn on the topographies in the same figure. From these line profiles, it is apparent
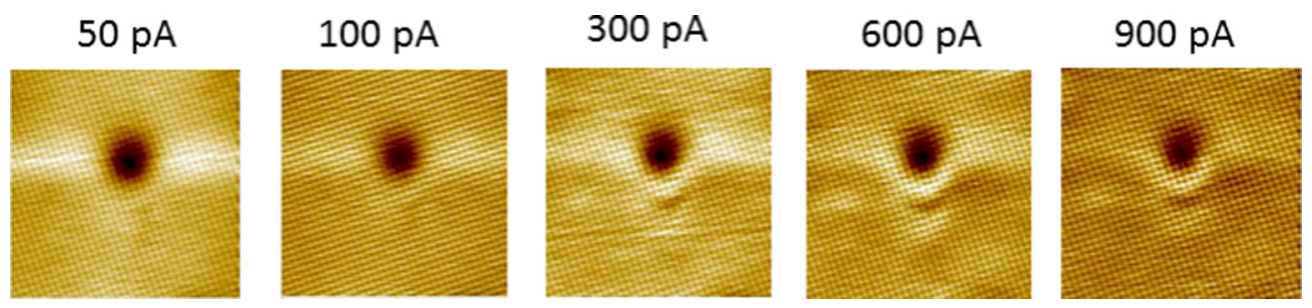

FIG. 9. STM topographies of $\mathrm{EuB}_{6}$ taken on a surface area of $15 \times 15 \mathrm{~nm}^{2}$ with a buried impurity at different tunneling currents. The bias voltage $V=-0.2 \mathrm{~V}$. 

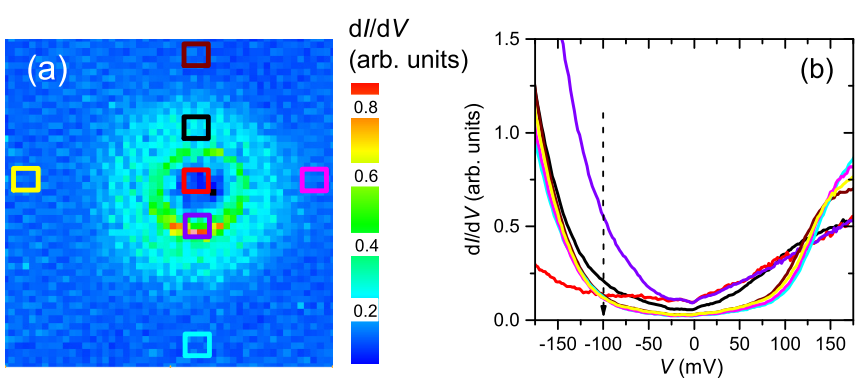

FIG. 10. (a) Conductance map $g(V, \vec{r})=d I(V, \vec{r}) / d V$ at $V=$ $-100 \mathrm{mV}$ taken on the topography shown in Fig. 6. (b) $d I(V) / d V$ spectra averaged within the areas marked by the same color in (a). The arrow indicates the value of bias voltage at which $g(V, \vec{r})$ in (a) is mapped.

that the sizes of the rings were found to be independent of $I_{\mathrm{sp}}$, even if the latter is varied by an order of magnitude. As mentioned in the main text, since the tunneling current falls off exponentially with respect to the tip-sample distance $d$, any tip-induced band bending effects (TIBB) should affect the size of the rings when $I_{\mathrm{sp}}$ is varied over a large range. These results suggest that TIBB is minimal in our samples. It is worthwhile to note here that in the case of the topological Kondo insulator $\mathrm{SmB}_{6}$, it has been concluded that the TIBB is insignificant [31].

We also performed local conductance maps $g(V, \vec{r})=$ $d I(V, \vec{r}) / d V$ in the bias voltage range -0.175 to $+0.175 \mathrm{mV}$ on the same area of topography shown in Fig. 6. As an example, a conductance map $g(V, \vec{r})$ at $V=-100 \mathrm{mV}$ is displayed in Fig. 10(a). As in the topography, a modulation was also observed in the spectroscopic map, which corresponds to an oscillation in the charge density around the impurity atom. The $d I / d V$ curves averaged over selected areas in Fig. 10(a) are plotted in Fig. 10(b). These spectra are specific to the particular surface shown in Fig. 6 and are markedly different from those reported in Ref. [7] for bulk $\mathrm{EuB}_{6}$. This implies that, in the present case, the tunneling matrix element is more sensitive to the $\Gamma$ point, where the system has a gap as can be seen in the bulk band structure [17].

In Fig. 11, we present details of the band structure near the Fermi edge for a slab $(N=12)$ with $\mathrm{B}_{6}$ termination on both sides, mainly to show the effects of hybridization described in the main text. Here the individual bands are singled out by different colors. The surface electronic state is composed of various individual bands, which are represented in the band dispersion by piecewise contributions of bands (apart of the double degeneracy for the top and bottom surface contribution). These complicated band-structure features of a finite slab reflect the fact that the surface electronic state is hybridized with extended bulk electronic states, when their energy dispersions match. This hybridization leads to resonances in the region where the bulk states are densely spaced around $\bar{\Gamma}$ and near $\bar{X}$, the underlying electronic bands then correspond to states, which are extended throughout the whole slab but with increased weight of the wave function near the surface. In the slab calculation, the continuum of the surface-projected bulk bands in resonance with the surface state is represented by a small countable set of such bands,
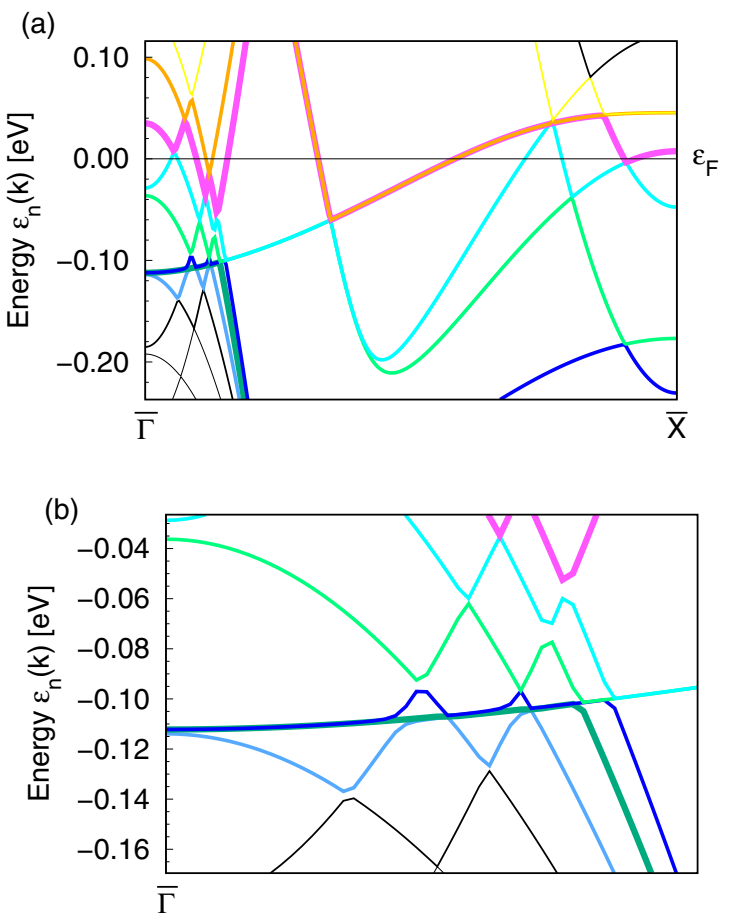

FIG. 11. Band structure details for $\mathrm{Eu}_{12} \mathrm{~B}_{(12+1) 6}$ slab with $\mathrm{B}_{6}$ termination on both surfaces. The individual bands near the Fermi energy $\varepsilon_{F}=0$ are plotted with different colors. The surface state is composed of piecewise contributions from various bands and also doubly degenerate from the contribution of top and bottom surface. (a) shows the cut for the surface Brillouin zone from $\bar{\Gamma}$ to $\overline{\mathrm{X}}$. (b) shows the details around $\bar{\Gamma}$.

as shown in the magnification Fig. 11(b). These bands do not cross, but repel each other and form small minigaps. The hybridized electronic states in this region around $\bar{\Gamma}$ represent surface-enhanced resonances of extended states.

Up to now, we presented details of localized perturbations on a (001) surface of $\mathrm{EuB}_{6}$ with $\mathrm{B}$ termination. A question naturally arises whether such effects occur also on the (001) surface with Eu termination. Experimentally, we rarely observed Eu-terminated surfaces (i.e., only a few patches on

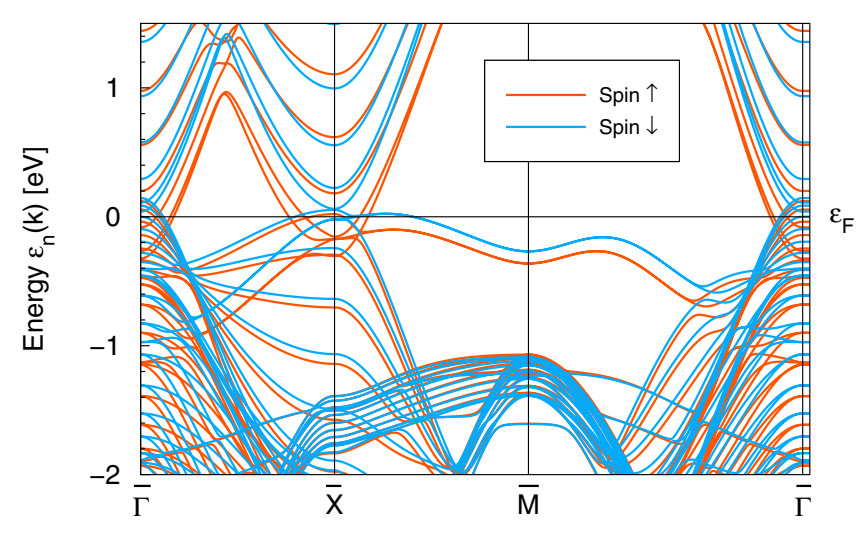

FIG. 12. The (001) surface band structure of Eu-terminated surface, $[\mathrm{EuB} 6]_{9} \mathrm{Eu}$ or $1 \times 1 \times 10$ supercell, calculated using GGA open core. 
two different cleaves). This is in line with our experience in the case of $\mathrm{SmB}_{6}$ where the observation of Sm-terminated surfaces was similarly rare. A recent soft x-ray reflectometry study on $\mathrm{SmB}_{6}$ has established that thorough a chemical surface reconstruction, a boron termination is eventually established, irrespective of the initial termination [38]. Similar chemical reconstructions may occur also in isostructural EuB $_{6}$. Nonetheless, we performed DFT calculations with the same method as for B-terminated surfaces also for the Eu- terminated ones (Fig. 12). Indeed, while there is a surfacerelated state in the corresponding band structures of these slabs, its location is much lower in energy, corresponding to an almost filled surface state. Also, the spin splitting of this surface is much smaller, in correspondence to the bulk states of $\mathrm{EuB}_{6}$. Therefore, the screening charge cloud around point defects in these surfaces is expected to behave very differently due to the lack of empty states available for electron scattering to take place.
[1] D. M. Goebel, Y. Hirooka, and T. A. Sketchley, Rev. Sci. Instrum. 56, 1717 (1985).

[2] A. S. Cameron, G. Friemel, and D. S. Inosov, Rep. Prog. Phys. 79, 066502 (2016).

[3] R. Lortz, Y. Wang, U. Tutsch, S. Abe, C. Meingast, P. Popovich, W. Knafo, N. Shitsevalova, Yu. B. Paderno, and A. Junod, Phys. Rev. B 73, 024512 (2006).

[4] C. N. Guy, S. von Molnár, J. Etourneau, and Z. Fisk, Solid State Commun. 33, 1055 (1980).

[5] P. Das, A. Amyan, J. Brandenburg, J. Müller, P. Xiong, S. von Molnár, and Z. Fisk, Phys. Rev. B 86, 184425 (2012).

[6] R. S. Manna, P. Das, M. de Souza, F. Schnelle, M. Lang, J. Müller, S. von Molnár, and Z. Fisk, Phys. Rev. Lett. 113, 067202 (2014).

[7] M. Pohlit, S. Rößler, Y. Ohno, H. Ohno, S. von Molnár, Z. Fisk, Jens Müller, and S. Wirth, Phys. Rev. Lett. 120, 257201 (2018).

[8] M. Dzero, K. Sun, V. Galitski, and P. Coleman, Phys. Rev. Lett. 104, 106408 (2010).

[9] T. Takimoto, J. Phys. Soc. Jpn. 80, 123710 (2011).

[10] S. Süllow, I. Prasad, M. C. Aronson, J. L. Sarrao, Z. Fisk, D. Hristova, A. H. Lacerda, M. F. Hundley, A. Vigliante, and D. Gibbs, Phys. Rev. B 57, 5860 (1998).

[11] S. Süllow, I. Prasad, M. C. Aronson, S. Bogdanovich, J. L. Sarrao, and Z. Fisk, Phys. Rev. B 62, 11626 (2000).

[12] R. R. Urbano, P. G. Pagliuso, C. Rettori, S. B. Oseroff, J. L. Sarrao, P. Schlottmann, and Z. Fisk, Phys. Rev. B 70, 140401(R) (2004)

[13] X. Zhang, L. Yu, S. von Molnár, Z. Fisk, and P. Xiong, Phys. Rev. Lett. 103, 106602 (2009).

[14] S. von Molnár, Sensors \& Actuators A: 91, 161 (2001).

[15] P. Nyhus, S. Yoon, M. Kauffman, S. L. Cooper, Z. Fisk, and J. Sarrao, Phys. Rev. B 56, 2717 (1997).

[16] M. J. Calderón, L. G. L. Wegener, and P. B. Littlewood, Phys. Rev. B 70, 092408 (2004).

[17] S. Massidda, A. Continenza, T. M. de Pascale, and R. Monnier, Z. Phys. B 102, 83 (1997).

[18] R. G. Goodrich, N. Harrison, J. J. Vuillemin, A. Teklu, D. W. Hall, Z. Fisk, D. Young, and J. Sarrao, Phys. Rev. B 58, 14896 (1998).

[19] M. C. Aronson, J. L. Sarrao, Z. Fisk, M. Whitton, and B. L. Brandt, Phys. Rev. B 59, 4720 (1999).

[20] J. D. Denlinger, J. A. Clack, J. W. Allen, G.-H. Gweon, D. M. Poirier, C. G. Olson, J. L. Sarrao, A. D. Bianchi, and Z. Fisk, Phys. Rev. Lett. 89, 157601 (2002).

[21] J. D. Denlinger, G.-H. Gweon, S.-K. Mo, J. W. Allen, J. L. Sarrao, A. D. Bianchi, and Z. Fisk, J. Phys. Soc. Jpn. 71, 1 (2002).
[22] J. Kuneš and W. E. Pickett, Phys. Rev. B 69, 165111 (2004).

[23] M. Kreissl and W. Nolting, Phys. Rev. B 72, 245117 (2005).

[24] X. Zhang, S. von Molnár, Z. Fisk, and P. Xiong, Phys. Rev. Lett. 100, 167001 (2008).

[25] M. M. Yee, Y. He, A. Soumyanarayanan, D.-J. Kim, Z. Fisk, and J. E. Hoffman, arXiv:1308.1085.

[26] W. Ruan, C. Ye, M. Guo, F. Chen, X. Chen, G.-M. Zhang, and Y. Wang, Phys. Rev. Lett. 112, 136401 (2014).

[27] S. Rößler, T.-H. Jang, D.-J. Kim, L. H. Tjeng, Z. Fisk, F. Steglich, and S. Wirth, Proc. Natl. Acad. Sci. USA 111, 4798 (2014).

[28] S. Rößler, L. Jiao, D. J. Kim, S. Seiro, K. Rasim, F. Steglich, L. H. Tjeng, Z. Fisk, and S. Wirth, Philos. Mag. 96, 3262 (2016).

[29] Z. Sun, A. Maldonado, W. S. Paz, D. S. Inosov, A. P. Schnyder, J. J. Palacios, N. Y. Shitsevalova, V. B. Filipov, and P. Wahl, Phys. Rev. B 97, 235107 (2018).

[30] L. Jiao, S. Rößler, D. Kasinathan, P. F. S. Rosa, C. Guo, H. Yuan, C.-X. Liu, Z. Fisk, F. Steglich, and S. Wirth, Sci. Adv. 4, eaau4886 (2018).

[31] H. Pirie, Y. Liu, A. Soumyanarayanan, P. Chen, Y. He, M. M. Yee, P. F. S. Rosa, J. D. Thompson, D.-J. Kim, Z. Fisk, X. Wang, J. Paglione, D. K. Morr, M. H. Hamidian, and J. E. Hoffman, Nature Phys. 16, 52 (2020)

[32] H. Herrmann, P. Hlawenka, K. Siemensmeyer, E. Weschke, J. Sánchez-Barriga, A. Varykhalov, N. Y. Shitsevalova, A. V. Dukhnenko, V. B. Filipov, S. Gabáni, K. Flachbart, O. Rader, M. Sterrer, and E. D. L. Rienks, Adv. Mater. 32, 1906725 (2020).

[33] J. D. Denlinger, APS March Meeting 2007, http://meetings.aps. org/link/BAPS.2007.MAR.A7.3.

[34] Z. Fisk, D. C. Johnston, B. Cornut, S. von Molnár, S. Oseroff, and R. Calvo, J. Appl. Phys. 50, 1911 (1979).

[35] K. Koepernik and H. Eschrig, Phys. Rev. B 59, 1743 (1999).

[36] J. P. Perdew, K. Burke, and M. Ernzerhof, Phys. Rev. Lett. 77, 3865 (1996).

[37] Y. Takahashi, M. Fujimoto, M. Tsuchikoa, and K.-I. Ohshimaa, J. Appl. Crystallogr. 34, 208 (2001).

[38] V. B. Zabolotnyy, K. Fürsich, R. J. Green, P. Lutz, K. Treiber, C.-H. Min, A. V. Dukhnenko, N. Y. Shitsevalova, V. B. Filipov, B. Y. Kang, B. K. Cho, R. Sutarto, Feizhou He, F. Reinert, D. S. Inosov, and V. Hinkov, Phys. Rev. B 97, 205416 (2018).

[39] K. Teichmann, M. Wenderoth, S. Loth, R. G. Ulbrich, J. K Garleff, A. P. Wijnheijmer, and P. M. Koenraad, Phys. Rev. Lett. 101, 076103 (2008).

[40] F. Marczinowski, J. Wiebe, F. Meier, K. Hashimoto, and R. Wiesendanger, Phys. Rev. B 77, 115318 (2008). 
[41] A. P. Wijnheijmer, J. K. Garleff, K. Teichmann, M. Wenderoth, S. Loth, and P. M. Koenraad, Phys. Rev. B 84, 125310 (2011).

[42] Y. Kohsaka, T. Hanaguri, M. Azuma, M. Takano, J. C. Davis, and H. Takagi, Nature Phys. 8, 534 (2012).

[43] I. Battisti, K. M. Bastiaans, V. Fedoseev, A. de la Torre, N. Iliopoulos, A. Tamai, E. C. Hunter, R. S. Perry, J. Zaanen, F. Baumberger, and M. P. Allan, Nature Phys. 13, 21 (2017).

[44] C.-L. Song, Y.-P. Jiang, Y.-L. Wang, Z. Li, L. Wang, K. He, X. Chen, X.-C. Ma, and Q.-K. Xue, Phys. Rev. B 86, 045441 (2012).

[45] I. Battisti, V. Fedoseev, K. M. Bastiaans, A. de la Torre, R. S. Perry, F. Baumberger, and M. P. Allan, Phys. Rev. B 95, 235141 (2017).
[46] M. C. M. M. van der Wielen, A. J. A. van Roij, and H. van Kempen, Phys. Rev. Lett. 76, 1075 (1996).

[47] R. Monnier and B. Delley, Phys. Rev. B 70, 193403 (2004).

[48] Z.-H. Zhu, A. Nicolaou, G. Levy, N. P. Butch, P. Syers, X. F. Wang, J. Paglione, G. A. Sawatzky, I. S. Elfimov, and A. Damascelli, Phys. Rev. Lett. 111, 216402 (2013).

[49] J. Kim, K. Kim, C. J. Kang, S. Kim, H. C. Choi, J. S. Kang, J. D. Denlinger, and B. I. Min, Phys. Rev. B 90, 075131 (2014).

[50] S. Nie, Y. Sun, F. B. Prinz, Z. Wang, H. Weng, Z. Fang, and X. Dai, Phys. Rev. Lett. 124, 076403 (2020).

[51] For a discussion on the open-core method for $4 f$-compounds, see M. Richter, J. Phys. D: Appl. Phys. 31, 1017 (1998). 